\title{
ON BIPOLAR JET FORMATION IN PLANETARY NEBULAE
}

\author{
G. PASCOLI \\ Faculté des Sciences, Department of Physics, Amiens, France
}

Bipolar outflows or polar knots are rather ubiquitous phenomena. A wealth of observational data is now available on bipolar flows associated with young stellar objects or stellar formation regions (Snell, Proc.I.A.U.Symp.Nr. 115, 1987), or nuclei of active galaxies (Asseo and Sol, 1987, Phys.Rep. 148, 307).

In the Planetary Nebula (PN) context, the origin of bipolar jets has never been completely elucidated (Morris, Proc.Coll. "From Miras to PNe", Montpellier, France, 1990); even though interesting suggestions have been made in this sense (Soker and Livio, 1989, ApJ 339, 268).

We propose here a stationary magnetohydrodynamic model in order to explain the origin of bipolar jets or highly collimated outflows in PNe. Analytical calculations have been performed taking into account the effects of both a large scale azimuthal magnetic field and an anisotropic turbulent velocity field within the gas.

A comparison with the observational data (polar-to-equatorial density contrast, polar velocity magnitude and isophotal maps) allows us to expect the equatorialto-polar density ratio to be typically in the range of $2-3$. On the other hand, the present model does predict polar jet velocities $\sim 100 \mathrm{~km}$ (s (assuming an equatorial velocity $\sim 10 \mathrm{~km} / \mathrm{s}$ ), in good agreement with the observations. Stemming from our hypotheses, another interesting conclusion is that elongated turbulent cells, similar to filamentary structures, very likely appear within the polar regions. On the other hand a ring of magnetized matter is also formed in the equatorial plane. Theoretical isophotes are produced.

The model can be applied to objects such as He 2-104, Mz3, M2-9. 